\title{
FRAÇÕES OXIDÁVEIS DO CARBONO ORGÂNICO DE LATOSSOLO CULTIVADO COM CAFEEIRO EM DIFERENTES ESPAÇAMENTOS DE PLANTIO ${ }^{1}$
}

\author{
Oxidizible organic carbon fractions in a latosol cultivated with coffee \\ at different planting spacings
}

\author{
Otacílio José Passos Rangel ${ }^{2}$, Carlos Alberto Silva ${ }^{3}$, Paulo Tácito Gontijo Guimarães ${ }^{4}$, \\ Luiz Roberto Guimarães Guilherme ${ }^{5}$
}

\begin{abstract}
RESUMO
Objetivou-se com este estudo avaliar a suscetibilidade à oxidação pelo permanganato de potássio do carbono presente em Latossolo Vermelho distroférrico típico, cultivado com cafeeiro (Coffea arabica L.), em diferentes espaçamentos de plantio, em experimento localizado na Fazenda Experimental da EPAMIG, em Machado (MG). Os tratamentos avaliados, instalados a campo em 1992, consistiram na combinação de quatro espaçamentos entre linhas $(2,0,2,5,3,0$ e 3,5 m), com três espaçamentos entre plantas $(0,5,0,75$ e $1,0 \mathrm{~m})$. Uma área de mata próxima ao experimento foi amostrada e usada como referência. Para a avaliação do grau de oxidação do carbono orgânico (CO), as amostras de solo foram coletadas na entrelinha (EL) e na projeção da copa (PC) do cafeeiro, nas camadas de solo de 0-0,05 e 0-0,1 m. Na camada superficial do solo (0-0,05 m), os teores de C lábil na entrelinha são, em geral, maiores do que os observados nas amostras coletadas na projeção da copa do cafeeiro. A amplitude de variação dos teores de $\mathrm{C}$ nas frações orgânicas obedece à seguinte ordem decrescente: $\mathrm{C}$ lábil > C não lábil > $\mathrm{C}$ orgânico, o que indica o maior potencial do $\mathrm{C}$ lábil em avaliar o impacto de diferentes sistemas de adensamento e manejo do cafeeiro, sobre os compartimentos de matéria orgânica. Os teores de C lábil e C não lábil não são influenciados pelo número de plantas por área, mas são dependentes da largura de rua do cafeeiro, da profundidade e do local de coleta das amostras de solo.
\end{abstract}

Termos para indexação: Coffea arabica L., densidade de plantio, matéria orgânica do solo, carbono lábil.

\section{ABSTRACT}

The present work was carried out to evaluate soil carbon susceptibility to oxidation by potassium permanganate in adystroferric Red Latosol (Oxisol) cultivated with coffee (Coffea arabica L.) at different planting spacings. The experiment was installed in 1992 at the Experimental Farm of EPAMIG, in Machado (Minas Gerais State-Brazil). Treatments consisted of a combination of four row spacings $(2.0,2.5,3.0$, and $3.5 \mathrm{~m})$ with three spacings within the row $(0.5,0.75$, and $1.0 \mathrm{~m})$. As a reference, soil samples were also collected in an area under native forest. For assessing the degree of oxidation of the organic carbon (OC), soil samples were collected at the inter-row and in the coffee canopy projection, at the 0-0.05 and 0-0.1 m soil depths. Labile $\mathrm{C}$ in inter-row samples located at the soil surface layer $(0-0.05 \mathrm{~m})$ is, in general, higher than that measured in samples collected in the coffee canopy projection. The amplitude of variation of $\mathrm{C}$ in different soil organic fractions follows the order: labile $\mathrm{C}>$ non labile $\mathrm{C}>$ Organic $\mathrm{C}$. Thus, labile $\mathrm{C}$ could be used as a sensitive index to evaluate the impact of different coffee planting systems and management practices on soil organic matter pools. Labile and non labile $\mathrm{C}$ contents are not influenced by the number of coffee plants per area. However, these indexes are dependent on coffee plants inter-row spacings, soil depth and sampling location.

Index terms: Coffea arabica L., planting density, soil organic matter, labile carbon.

(Recebido em 16 de abril de 2006 e aprovado em 11 de maio de 2007)

\section{INTRODUÇÃO}

A intervenção humana nos ecossistemas naturais para a implantação de atividades agropecuárias diminui os estoques e altera a composição química da matéria orgânica do solo (MOS) (SILVA et al., 1999). No estudo desses impactos, os sistemas de produção com culturas anuais são os mais investigados, em virtude da sua grande extensão, histórico de uso mais antigo e importância econômica. Os resultados desses estudos indicam que as

\footnotetext{
${ }^{1}$ Parte da Tese de Doutorado do primeiro autor desenvolvida no Departamento de Ciência do Solo da Universidade Federal de Lavras/UFLA. ${ }^{2}$ Doutor em Agronomia - INCRA - Rua Rubens de Mendonça, 652/1202 - Baú - 78008-000 - Cuiabá, MT - otaciliorangel@yahoo.com.br ${ }^{3}$ Doutor, Professor Adjunto - Departamento de Ciência do Solo/DCS - Universidade Federal de Lavras/UFLA - Cx. P. 3037 - $37200-000$ - Lavras, MG csilva@ufla.br

${ }^{4}$ Doutor em Agronomia, Pesquisador - Empresa de Pesquisa Agropecuária de Minas Gerais/EPAMIG - CX. P. 176 - $37200-000$ - Lavras, MG paulotgg@ufla.br

${ }^{5} \mathrm{Ph}$. D. em Agronomia/Química Ambiental - Departamento de Ciência do Solo/DCS - Universidade Federal de Lavras/UFLA - Cx. P. 3037- 37200-000 Lavras, MG - guilherm@ufla.br
} 
perdas de matéria orgânica do solo (MOS) chegam a mais de $50 \%$ dos teores iniciais, em períodos relativamente curtos (menos de 10 anos), especialmente nos solos de textura mais arenosa e onde as práticas de manejo do solo são menos conservacionistas (MIELNICZUK et al., 2003). Entretanto, poucos são os estudos onde foi avaliado o impacto do cultivo de espécies perenes sobre os teores e composição da MOS.

No estado de Minas Gerais, uma das culturas perenes com maior expressão econômica é a do café, cuja expansão tem sido substancial nas últimas décadas, elevando o estado à categoria de maior produtor do país. Como resultado disso, observa-se que, dos 20 municípios maiores produtores de café do Brasil, oito estão localizados no estado de Minas Gerais (ABIC, 2006).

Nos últimos anos, a cafeicultura mineira tem passado por grandes alterações no sistema de cultivo, principalmente em relação à densidade populacional. Os espaçamentos largos, característicos da cafeicultura tradicional, estão sendo substituídos por espaçamentos menores, comumente conhecidos como sistema adensado (PAVAN \& CHAVES, 1996). Além do aumento na produção, o sistema adensado proporciona maior proteção do solo, constituindo-se em importante prática de manejo para a recuperação dos teores de MOS. Pavan et al. (1999) observaram que o aumento do número de plantas, em lavoura cafeeira de 893 para 7.143 plantas por hectare resultou em um acréscimo de $36 \%$ no teor de carbono orgânico $(\mathrm{CO})$ do solo, na profundidade de $0-20 \mathrm{~cm}$. Paralelamente ao aumento no teor de carbono orgânico, os autores também relataram, com o adensamento da lavoura cafeeira, alterações nas principais características químicas do solo, como aumento do $\mathrm{pH}$, da capacidade de troca de cátions (CTC), dos teores de $\mathrm{Ca}^{2+}, \mathrm{Mg}^{2+}, \mathrm{P}^{2} \mathrm{~K}^{+} \mathrm{e}$ diminuição dos teores de $\mathrm{Al}^{3+}$.

Além das mudanças na quantidade de matéria orgânica em solos cultivados, também têm sido observadas mudanças na sua qualidade, notadamente no grau de oxidação e labilidade (BLAIR et al., 1995; SHANG \& TIESSEN, 1997). Em regiões de clima temperado, o cultivo contínuo do solo aumenta o grau de aromaticidade da matéria orgânica e diminui a presença de frações orgânicas de maior biodisponibilidade (PRESTON, 1996). Em solos tropicais, naturalmente, a biodisponibilidade da matéria orgânica é menor, sendo elevada a presença de compostos orgânicos ricos em grupamentos aromáticos e carboxílicos (ZECH et al., 1990). Em alguns solos brasileiros, estudos têm mostrado que as perdas acentuadas de carbono com o desmatamento e cultivo dos solos são acompanhadas pelo consumo de frações orgânicas de maior labilidade, o que implica em aumento do já elevado grau de aromaticidade da MOS (SILVA et al., 1994, 1999). As reduções nos estoques de $\mathrm{C}$ e as mudanças na composição da matéria orgânica comprometem a sustentabilidade desses agroecossistemas, uma vez que a maior disponibilidade, no solo, de compostos orgânicos pode aumentar a CTC (BAYER \& BERTOL, 1999), a atividade microbiana (SCHERER et al., 2007), diminuir os efeitos negativos do alumínio tóxico (CIOTTA et al., 2002) e reduzir a adsorção de grupamentos fosfatos aos colóides dos solos (GUERTAL et al., 1991).

Conceitualmente, carbono lábil é aquele constituinte de compostos orgânicos mais facilmente mineralizado pelos microrganismos do solo, sendo que procedimentos colorimétricos baseados na oxidação do carbono com permanganato de potássio $\left(\mathrm{KMnO}_{4}\right)$ têm sido utilizados para diferenciar a labilidade do C. Blair et al. (1995) consideram C lábil o carbono oxidável por uma solução de permanganato de potássio $\left(\mathrm{KMnO}_{4}\right) \quad 0,333 \mathrm{~mol} \mathrm{~L}^{-1}$. Entretanto, Shang \& Tiessen (1997) propuseram a diminuição da concentração de $\mathrm{KMnO}_{4}$ para $0,033 \mathrm{~mol} \mathrm{~L}^{-1}$, considerada suficiente para oxidar o C lábil do solo. Em solos tropicais, o emprego de soluções com menor poder de oxidação do que a utilizada por Blair et al. (1995) se justifica pelo fato de estudos de datação utilizando ${ }^{14} \mathrm{C}$ mostrarem que a matéria orgânica de solos tropicais é mais lábil do que a presente em solos de regiões de clima temperado (SHANG \& TIESSEN, 1997).

Com o objetivo de avaliar diferentes frações de oxidação do C do solo, Chan et al. (2001) introduziram uma modificação no método clássico de determinação do C desenvolvido por Walkley \& Black (1934). No método original, o $\mathrm{C}$ era determinado pela utilização de uma única concentração de ácido sulfúrico $\left(12 \mathrm{~mol} \mathrm{~L}^{-1}\right)$ e, com a modificação proposta por Chan et al. (2001), foi possível separar quatro frações com graus decrescentes de oxidação, por meio da utilização de quantidades crescentes de ácido sulfúrico. Um entrave ao uso dessa metodologia é a dificuldade de se comparar os valores obtidos com os determinados pelos métodos descritos por Blair et al. (1995) e Shang \& Tiessen (1997), pois é evidente que, com a mudança nas concentrações de ácidos e dos oxidantes, determinadas formas de carbono, antes não afetadas, serão oxidadas pela solução oxidante proposta por Chan et al. (2001).

Nos estudos desenvolvidos por Blair et al. (1995), o carbono lábil representou aproximadamente $50 \%$ do CO do solo, enquanto que nos estudos de Shang \& Tiessen (1997), com a utilização de uma menor concentração de $\mathrm{KMnO}_{4}$, o carbono lábil representou, em média, $25 \%$ do 
CO. Com base no procedimento descrito por Chan et al. (2001), Freitas et al. (2004), avaliando o impacto da adubação orgânica e mineral sobre a qualidade da MOS, encontraram valores de carbono lábil correspondendo a $28 \%$ do $\mathrm{CO}$ do solo. Já Andrade et al. (2005) observaram que 50\% do C na MOS de solo adubado com lodo de esgoto foi classificado como C lábil. Dessa forma, a separação do C em diferentes graus de oxidação requer ajustes metodológicos, além das mudanças necessárias quanto aos aspectos relacionados às diferenças de clima, manejo e solo, que alteram as taxas de oxidação da MOS.

A ausência de resultados ligados ao efeito de sistemas de cultivo do cafeeiro sobre o grau de oxidação da MOS é um dos aspectos que justificam este estudo. Avaliações dessa natureza são essenciais não apenas para averiguar o impacto da interferência da atividade no ecossistema, mas também para determinar se a qualidade do solo em áreas cafeeiras não está sendo comprometida em médio e longo prazos, em função da degradação e perda acelerada da MOS. Objetivou-se neste estudo avaliar o grau de oxidação do carbono orgânico de um Latossolo Vermelho distroférrico cultivado com cafeeiro, em diferentes espaçamentos de plantio, no sul do estado de Minas Gerais.

\section{MATERIAL E MÉTODOS}

O estudo foi conduzido na Fazenda Experimental da Empresa de Pesquisa Agropecuária de Minas Gerais (EPAMIG), localizada no município de Machado (MG). O solo da área experimental foi classificado como Latossolo Vermelho distroférrico típico, textura média. As principais características químicas e físicas do solo $(0-20 \mathrm{~cm})$, referentes às amostras coletadas antes da implantação do experimento a campo, são: $\mathrm{pH}$ em água $=4,0, \mathrm{Ca}^{2+}=0,2$ cmol $\mathrm{kg}^{-1}, \mathrm{Mg}^{2+}=0,1 \mathrm{cmol}_{\mathrm{c}} \mathrm{kg}^{-1}, \mathrm{Al}^{3+}=0,5 \mathrm{cmol} \mathrm{kg} \mathrm{kg}^{-1}, \mathrm{P}$ (Mehlich-1) $=1,0 \mathrm{mg} \mathrm{kg}^{-1}, \mathrm{~K}^{+}=11 \mathrm{mg} \mathrm{kg}^{-1}$, areia $=440 \mathrm{~g} \mathrm{~kg}^{-1}$, silte $=290 \mathrm{~g} \mathrm{~kg}^{-1}$, argila $=270 \mathrm{~g} \mathrm{~kg}^{-1}$.

A instalação do experimento a campo, caracterizada pelo plantio das mudas do cafeeiro, foi realizada em janeiro de 1992, sulcando-se o solo de acordo com os espaçamentos estudados, utilizando-se no plantio a cultivar Catuaí Vermelho IAC-44, com uma planta por cova. Durante a condução do experimento (1992 a 2003), o cafeeiro foi mantido livre de plantas invasoras, por meio de capinas manuais ( 5 a 6 capinas por ano) e aplicações de herbicidas. Os restos culturais do cafeeiro e de ervas daninhas, em função das práticas de cultivo e arruação, foram depositados nas entrelinhas de plantio, de modo que, na projeção da copa, o solo foi mantido sem cobertura vegetal durante a maior parte do ano.
Os tratamentos avaliados consistiram da combinação de quatro espaçamentos entrelinhas $(2,0 ; 2,5$; $3,0$ e $3,5 \mathrm{~m})$ com três espaçamentos entre plantas $(0,5$; 0,75 e $1,0 \mathrm{~m}$ ) de cafeeiro, totalizando 12 tratamentos, dispostos no campo em blocos ao acaso, em esquema fatorial 4x3, com três repetições. As parcelas experimentais mediam doze metros de comprimento e possuíam três linhas de plantio, cujas distâncias entre si variaram em função do tratamento estudado. A parcela útil considerada na etapa de coleta das amostras de solo foi a linha central (interna), uma vez que ela sofreu a influência tanto do adensamento entrelinhas, como entre plantas na linha. A densidade populacional de cafeeiro variou, em função dos tratamentos estudados, de 2.857 a 10.000 plantas por hectare.

A amostragem do solo foi realizada em dezembro de 2003, onze anos após o estabelecimento do experimento a campo. As amostras foram coletadas em dois pontos de cada parcela experimental, na projeção da copa (PC) e na entrelinha de cultivo (EL), onde eram depositados, antes das colheitas, os resíduos culturais e os provenientes das capinas da PC. Foram retiradas amostras compostas nas profundidades de 0-0,05 e 0-0,1 $\mathrm{m}$, coletando-se cinco amostras simples em cada ponto (EL e PC), perfazendo uma amostra composta por parcela. Numa área sob mata, a cerca de um quilômetro do experimento, com a mesma classe de solo e sem histórico de intervenção humana, foram coletadas amostras de solo nas mesmas profundidades coletadas na área experimental, que foram utilizadas com referência de área sob vegetação natural. As amostras de solo foram homogeneizadas, acondicionadas em sacos plásticos e conduzidas para o laboratório, onde foram secadas ao ar, destorroadas, trituradas em almofariz e passadas em peneira de malha de $0,21 \mathrm{~mm}$.

$\mathrm{O}$ método utilizado para o fracionamento do $\mathrm{C}$ por graus de oxidação foi adaptado de Chan et al. (2001) e Freitas et al. (2004). Amostras de 0,3 g de solo foram acondicionadas em frasco erlenmeyer de $250 \mathrm{~mL}$, onde adicionaram-se $10 \mathrm{~mL} \mathrm{~K}_{2} \mathrm{Cr}_{2} \mathrm{O}_{7} 0,167 \mathrm{~mol} \mathrm{~L}^{-1}$ e quantidades de $\mathrm{H}_{2} \mathrm{SO}_{4}$ p.a., correspondentes às concentrações de 3, 6, 9 e $12 \mathrm{~mol} \mathrm{~L}^{-1}$. A oxidação foi realizada sem fonte externa de calor e a titulação dos extratos foi feita com uma solução de $\mathrm{Fe}\left(\mathrm{NH}_{4}\right)_{2}\left(\mathrm{SO}_{4}\right)_{2} \cdot 6 \mathrm{H}_{2} \mathrm{O} 0,4 \mathrm{~mol} \mathrm{~L}^{-1}$ (sal de Mohr), utilizando-se como indicador a fenantrolina $\left(\mathrm{C}_{12} \mathrm{H}_{8} \mathrm{~N}_{2} \mathrm{H}_{2} \mathrm{O}\right)$, preparada em função da mistura de $1,465 \mathrm{~g}$ de indicador com $0,985 \mathrm{~g}$ de $\mathrm{Fe}\left(\mathrm{NH}_{4}\right)_{2}\left(\mathrm{SO}_{4}\right)_{2} \cdot 6 \mathrm{H}_{2} \mathrm{O}$, que foram dissolvidos em $100 \mathrm{~mL}$ de água destilada. $\mathrm{O}$ fracionamento do $\mathrm{C}$ produziu quatro frações, com graus decrescentes de oxidação: 
- Fração $1\left(\mathrm{~F}_{1}\right)$ : C oxidado por $\mathrm{K}_{2} \mathrm{Cr}_{2} \mathrm{O}_{7}$ em meio ácido de $3 \mathrm{~mol} \mathrm{~L}^{-1}$ de $\mathrm{H}_{2} \mathrm{SO}_{4}$.

- Fração $2\left(\mathrm{~F}_{2}\right)$ : diferença do $\mathrm{C}$ oxidado por $\mathrm{K}_{2} \mathrm{Cr}_{2} \mathrm{O}_{7}$ em meio ácido com 6 e $3 \mathrm{~mol} \mathrm{~L}^{-1} \mathrm{de}_{2} \mathrm{SO}_{4}$.

- Fração $3\left(\mathrm{~F}_{3}\right)$ : diferença do $\mathrm{C}$ oxidado por $\mathrm{K}_{2} \mathrm{Cr}_{2} \mathrm{O}_{7}$ em meio ácido com 9 e $6 \mathrm{~mol} \mathrm{~L}^{-1}$ de $\mathrm{H}_{2} \mathrm{SO}_{4}$.

- Fração $4\left(\mathrm{~F}_{4}\right)$ : diferença do $\mathrm{C}$ oxidado por $\mathrm{K}_{2} \mathrm{Cr}_{2} \mathrm{O}_{7}$ em meio ácido com 12 e $9 \mathrm{~mol} \mathrm{~L}^{-1} \mathrm{de}_{2} \mathrm{SO}_{4}$.

$\mathrm{O}$ teor de $\mathrm{C}$ na fração $\mathrm{F}_{1}$ foi considerado como carbono lábil $\left(\mathrm{C}_{\mathrm{L}}\right)$ do solo, enquanto que o carbono nãolábil $\left(\mathrm{C}_{\mathrm{NL}}\right)$ foi obtido por diferença $\left(\mathrm{C}_{\mathrm{NL}}=\mathrm{CO}-\mathrm{C}_{\mathrm{L}}\right)$. Os teores de carbono orgânico $(\mathrm{CO})$ do solo foram determinados pela metodologia da combustão úmida com dicromato de potássio, descrita em Yeomans \& Bremner (1988) e utilizados no cálculo da relação $C_{L} / C O$, do Índice de Compartimento de Carbono (ICC) e na identificação de frações da MOS mais sensíveis às mudanças causadas pela adoção dos diferentes espaçamentos de plantio do cafeeiro. Tendo como base as variações nos teores de CO do solo da área de referência (mata nativa) e do solo cultivado com cafeeiro, foram calculados os seguintes índices: $(\mathrm{ICC})=\mathrm{CO}_{\text {cultivado }} / \mathrm{CO}_{\text {referência }}$, Labilidade $(\mathrm{L})=\mathrm{C}_{\mathrm{L}} /$ $\mathrm{C}_{\mathrm{NL}}$, Índice de Labilidade (IL) $=\mathrm{L}_{\text {cultivado }} / \mathrm{L}_{\text {referência }}$ e o Índice de Manejo de Carbono (IMC) = ICC x IL x 100, de acordo com Blair et al. (1995).

Os resultados obtidos foram submetidos à análise de variância para verificação, em cada profundidade de solo, do efeito dos diferentes espaçamentos de plantio e pontos de coleta das amostras de solo (EL e PC), sobre as frações do carbono orgânico com graus variáveis de oxidação. Os dados levantados na área de mata foram excluídos da análise estatística pelo fato de o local não compor o desenho experimental usado para avaliar o efeito dos espaçamentos de plantio de cafeeiro, sobre os atributos estudados. As comparações das médias foram feitas pelo teste de Scott-Knott, a $5 \%$ de probabilidade.

\section{RESULTADOS E DISCUSSÃO}

Os dados referentes ao fracionamento do $\mathrm{CO}$, em função da suscetibilidade das frações orgânicas à oxidação por permanganato de potássio são apresentados na Tabela 1. Para todos os espaçamentos de plantio e pontos de coleta das amostras de solo, a maior proporção do $\mathrm{C}$ esteve associada às frações de oxidação $\mathrm{F}_{1}$ e $\mathrm{F}_{2}$, o que implica em uma maior predominância, no solo sob cultivo de cafeeiro, de matéria orgânica de maior biodisponibilidade. $\mathrm{O}$ teor de carbono lábil $\left(\mathrm{C}_{\mathrm{L}}\right)$ na EL foi, em média, $43 \%$ (0-0,05 m) e 18\% $(0-0,1 \mathrm{~m})$ superior aos obtidos nas amostras coletadas na PC e $29 \%$ (0-0,05 m) maior, em relação ao solo de mata. Os resultados também indicam a redução nos teores de $C_{L}$, com o aumento da profundidade do solo, resultados também observados por Andrade et al. (2005). De modo geral, os resultados de pesquisas com frações de oxidação do $\mathrm{C}$ evidenciam que culturas agrícolas e, ou, sistemas de manejo que favorecem adições freqüentes de material orgânico ao solo, tendem a apresentar maior proporção de carbono na fração lábil, em detrimento das frações mais resistentes à oxidação (BLAIR et al., 1995; CHAN et al., 2001); isso de fato está de acordo com o histórico das parcelas experimentais avaliadas, na medida em que houve maior deposição de resíduos na EL do cafeeiro e no solo de mata, em relação às amostras coletadas na projeção da copa do cafeeiro.

Comparando-se os diversos espaçamentos de plantio do cafeeiro, é possível notar que os maiores teores de $C_{L}$ são, em geral, verificados nos maiores espaçamentos entrelinhas (3,0 e 3,5 metros), que apresentavam a maior incidência de plantas invasoras nas ruas de cafeeiro (Tabela 1). Foi observada uma maior proporção na área de mata do $C$ nas frações $\mathrm{F}_{3}$ e $\mathrm{F}_{4}(32,8$ e $36,3 \%$, respectivamente, para as profundidades de 0-0,05 e 0-0,1 m), em relação à área de cultivo de cafeeiro. Isso evidencia o acúmulo nesse local de compostos orgânicos de maior estabilidade química e alto peso molecular, oriundos da decomposição e humificação da MOS (STEVENSON, 1994). A fração mais resistente $\left(\mathrm{F}_{4}\right)$ é denominada de "compartimento passivo" do C, nos modelos de simulação da dinâmica da MOS, com um tempo de reciclagem de até 2000 anos (CHAN et al., 2001).

Os teores de $\mathrm{C}_{\mathrm{L}}$ nas duas profundidades de solo avaliadas responderam por cerca de $44 \%$ do total de CO presente no solo, com destaque para o espaçamento $3 \times 1$ $\mathrm{m}$, na profundidade de $0-0,05 \mathrm{~m}$, onde $53,5 \%$ do $\mathrm{CO}$ do solo era representado pela fração lábil - C-F1 (Tabela 2). Os maiores valores de labilidade (L) e do índice de labilidade (IL) foram observados nos espaçamentos $2,5 \times 0,5,2,5 \times 0,75$, $3 \times 0,5$ e $3 \times 0,75 \mathrm{~m}$ (EL e PC), 3x1 m (EL) e, 2,5x1 m (PC) na profundidade de $0-5 \mathrm{~cm}$ e, 2x0,5 m (EL e PC), 2,5x1 m (EL) e, $2 \times 1$ e 2,5x0,75 m (PC) na profundidade de $0-10 \mathrm{~cm}$ (Tabela 2), o que implica, nesses espaçamentos, numa maior proporção de $\mathrm{C}_{\mathrm{L}}$ em relação ao solo de mata, e em um aumento da biodisponibilidade do C. Os índices de compartimento do carbono (ICC), apresentados na Tabela 2 , indicam que, em relação à referência (mata), as amostras coletadas na EL do cafeeiro apresentaram um menor decréscimo relativo nos teores de $\mathrm{CO}$, enquanto que, nas amostras coletadas na PC, onde o acúmulo de resíduos era reduzido e a intervenção antrópica era maior, os teores de $\mathrm{CO}$ foram reduzidos, ou seja, os valores de ICC são menores que 1 . 


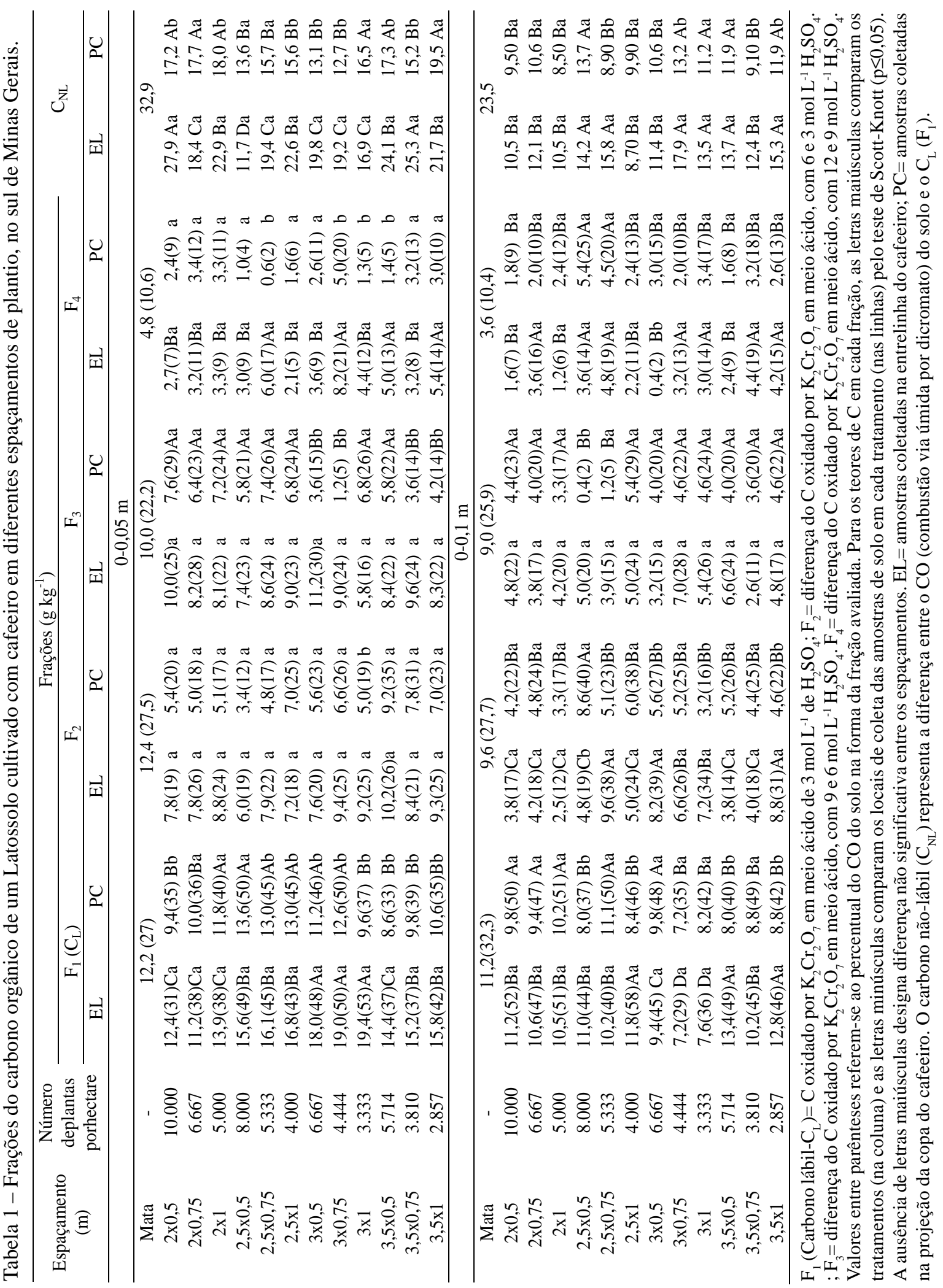

Ciênc. agrotec., Lavras, v. 32, n. 2, p. 429-437, mar./abr., 2008 
Tabela 2-Proporção de carbono lábil/carbono orgânico $\left(\mathrm{C}_{\mathrm{L}} / \mathrm{CO}\right)$, índice de compartimento de carbono (ICC), labilidade (L), índice de labilidade (IL) e índice de manejo de carbono (IMC), nas profundidades de 0-0,05 e 0-0,1 m de Latossolo Vermelho distroférrico típico, cultivado com cafeeiro em diferentes espaçamentos de plantio, no sul de Minas Gerais.

\begin{tabular}{|c|c|c|c|c|c|c|c|c|c|c|}
\hline \multirow{3}{*}{$\begin{array}{l}\text { Espaçamento, } \mathrm{m} \\
\text { (num. de plantas } \\
\text { por hectare) }\end{array}$} & \multirow{2}{*}{\multicolumn{2}{|c|}{$\mathrm{C}_{\mathrm{L}} / \mathrm{CO}(\%)$}} & \multicolumn{8}{|c|}{ Índice } \\
\hline & & & \multicolumn{2}{|c|}{ ICC } & \multicolumn{2}{|c|}{$\mathrm{L}$} & \multicolumn{2}{|c|}{ IL } & \multicolumn{2}{|c|}{ IMC } \\
\hline & EL & $\mathrm{PC}$ & EL & $\mathrm{PC}$ & EL & $\mathrm{PC}$ & EL & $\mathrm{PC}$ & EL & $\mathrm{PC}$ \\
\hline & \multicolumn{10}{|c|}{$0-0,05 \mathrm{~m}$} \\
\hline Mata & \multicolumn{2}{|c|}{$27,1( \pm 1,6)$} & \multicolumn{2}{|c|}{-} & \multicolumn{2}{|c|}{$0,37( \pm 0,03)$} & \multicolumn{2}{|c|}{ - } & \\
\hline $2 \times 0,5(10.000)$ & $30,8 \mathrm{Ba}$ & $35,7 \mathrm{Ba}$ & 0,89Aa & $0,59 \mathrm{Ab}$ & $0,45 \mathrm{Ba}$ & $0,58 \mathrm{Ba}$ & $1,20 \mathrm{Ba}$ & $1,56 \mathrm{Ba}$ & $107 \mathrm{Ba}$ & $90 \mathrm{Ba}$ \\
\hline $2 \times 0,75$ (6.667) & $38,0 \mathrm{Ba}$ & $36,0 \mathrm{Ba}$ & $0,66 \mathrm{Ba}$ & $0,62 \mathrm{Aa}$ & $0,61 \mathrm{Ba}$ & $0,57 \mathrm{Ba}$ & $1,65 \mathrm{Ba}$ & $1,52 \mathrm{Ba}$ & $108 \mathrm{Ba}$ & $94 \mathrm{Ba}$ \\
\hline $2 \times 1(5.000)$ & $37,8 \mathrm{Ba}$ & $40,0 \mathrm{Ba}$ & $0,81 \mathrm{Aa}$ & $0,66 \mathrm{Ab}$ & $0,62 \mathrm{Ba}$ & $0,67 \mathrm{Ba}$ & $1,66 \mathrm{Ba}$ & $1,81 \mathrm{Ba}$ & $134 \mathrm{Ba}$ & $117 \mathrm{Ba}$ \\
\hline $2,5 \times 0,5(8.000)$ & $49,7 \mathrm{Aa}$ & $50,0 \mathrm{Aa}$ & $0,61 \mathrm{Ba}$ & $0,60 \mathrm{Aa}$ & $1,00 \mathrm{Aa}$ & $1,00 \mathrm{Aa}$ & $2,73 \mathrm{Aa}$ & $2,70 \mathrm{Aa}$ & $162 \mathrm{Ba}$ & $163 \mathrm{Aa}$ \\
\hline $2,5 \times 0,75(5.333)$ & $45,4 \mathrm{Aa}$ & $45,4 \mathrm{Aa}$ & 0,79Aa & $0,64 \mathrm{Ab}$ & $0,84 \mathrm{Aa}$ & $0,83 \mathrm{Aa}$ & $2,25 \mathrm{Aa}$ & $2,24 \mathrm{Aa}$ & $178 \mathrm{Ba}$ & $142 \mathrm{Aa}$ \\
\hline $2,5 \times 1(4.000)$ & $38,5 \mathrm{Ba}$ & $45,4 \mathrm{Aa}$ & $0,87 \mathrm{Aa}$ & $0,63 \mathrm{Ab}$ & $0,62 \mathrm{Ba}$ & $0,84 \mathrm{Aa}$ & $1,68 \mathrm{Ba}$ & $2,27 \mathrm{Aa}$ & $146 \mathrm{Ba}$ & 144Aa \\
\hline $3 \times 0,5(6.667)$ & $47,3 \mathrm{Aa}$ & 45,9Aa & $0,84 \mathrm{Aa}$ & $0,54 \mathrm{Ab}$ & $0,90 \mathrm{Aa}$ & $0,85 \mathrm{Aa}$ & $2,42 \mathrm{Aa}$ & 2,29Aa & $205 \mathrm{Aa}$ & $124 \mathrm{Ab}$ \\
\hline $3 \times 0,75(4.444)$ & 49,9Aa & 49,7Aa & $0,85 \mathrm{Aa}$ & $0,56 \mathrm{Ab}$ & $1,01 \mathrm{Aa}$ & $1,01 \mathrm{Aa}$ & $2,72 \mathrm{Aa}$ & $2,71 \mathrm{Aa}$ & 229Aa & $153 \mathrm{Ab}$ \\
\hline $3 \times 1(3.333)$ & $53,5 \mathrm{Aa}$ & $36,8 \mathrm{Bb}$ & $0,80 \mathrm{Aa}$ & $0,58 \mathrm{Ab}$ & $1,16 \mathrm{Aa}$ & $0,60 \mathrm{Bb}$ & $3,12 \mathrm{Aa}$ & $1,61 \mathrm{Bb}$ & $251 \mathrm{Aa}$ & $93 \mathrm{Bb}$ \\
\hline $3,5 \times 0,5(5.714)$ & $37,4 \mathrm{Ba}$ & $33,3 \mathrm{Ba}$ & $0,85 \mathrm{Aa}$ & $0,57 \mathrm{Ab}$ & $0,60 \mathrm{Ba}$ & $0,51 \mathrm{Ba}$ & $1,61 \mathrm{Ba}$ & $1,37 \mathrm{Ba}$ & $138 \mathrm{Ba}$ & $78 \mathrm{Bb}$ \\
\hline $3,5 \times 0,75(3.810)$ & $37,6 \mathrm{Ba}$ & $39,5 \mathrm{Ba}$ & $0,90 \mathrm{Aa}$ & $0,55 \mathrm{Ab}$ & $0,60 \mathrm{Ba}$ & $0,66 \mathrm{Ba}$ & $1,62 \mathrm{Ba}$ & $1,76 \mathrm{Ba}$ & $145 \mathrm{Ba}$ & $97 \mathrm{Bb}$ \\
\hline \multirow[t]{2}{*}{$3,5 \times 1(2.857)$} & $42,1 \mathrm{Ba}$ & $35,3 \mathrm{Ba}$ & $0,83 \mathrm{Aa}$ & $0,67 \mathrm{Ab}$ & $0,74 \mathrm{Ba}$ & $0,55 \mathrm{Ba}$ & $2,00 \mathrm{Ba}$ & $1,47 \mathrm{Ba}$ & $166 \mathrm{Ba}$ & $98 \mathrm{Bb}$ \\
\hline & \multicolumn{10}{|c|}{$0-0,1 \mathrm{~m}$} \\
\hline Mata & \multicolumn{2}{|c|}{$32,3( \pm 1,5)$} & & \multicolumn{2}{|c|}{$0,48( \pm 0,03)$} & \multicolumn{2}{|c|}{ t } & \\
\hline $2 \times 0,5(10.000)$ & $51,7 \mathrm{Aa}$ & $50,9 \mathrm{Aa}$ & $0,63 \mathrm{Ba}$ & $0,56 \mathrm{Aa}$ & $1,07 \mathrm{Ba}$ & $1,05 \mathrm{Aa}$ & $2,26 \mathrm{Aa}$ & $2,20 \mathrm{Aa}$ & $140 \mathrm{Aa}$ & $122 \mathrm{Aa}$ \\
\hline $2 \times 0,75(6.667)$ & 47,1Aa & 47,0Aa & $0,65 \mathrm{Ba}$ & $0,58 \mathrm{Aa}$ & $0,91 \mathrm{Ca}$ & 0,89Ba & $1,92 \mathrm{Aa}$ & $1,87 \mathrm{Ba}$ & $123 \mathrm{Ba}$ & $107 \mathrm{Ba}$ \\
\hline $2 \times 1(5.000)$ & $49,7 \mathrm{Aa}$ & $54,6 \mathrm{Aa}$ & $0,60 \mathrm{Ba}$ & $0,54 \mathrm{Aa}$ & $0,99 \mathrm{Ba}$ & $1,20 \mathrm{Aa}$ & $2,10 \mathrm{Aa}$ & $2,53 \mathrm{Aa}$ & $125 \mathrm{Ba}$ & $136 \mathrm{Aa}$ \\
\hline $2,5 \times 0,5(8.000)$ & $43,7 \mathrm{Aa}$ & $36,9 \mathrm{Ba}$ & $0,73 \mathrm{Aa}$ & $0,63 \mathrm{Ab}$ & $0,78 \mathrm{Ca}$ & $0,59 \mathrm{Ba}$ & $1,64 \mathrm{Aa}$ & $1,24 \mathrm{Ba}$ & $119 \mathrm{Ba}$ & $77 \mathrm{Bb}$ \\
\hline $2,5 \times 0,75(5.333)$ & $39,2 \mathrm{Bb}$ & $56,3 \mathrm{Aa}$ & $0,75 \mathrm{Aa}$ & $0,58 \mathrm{Ab}$ & $0,65 \mathrm{Cb}$ & $1,34 \mathrm{Aa}$ & $1,36 \mathrm{Bb}$ & $2,82 \mathrm{Aa}$ & $102 \mathrm{Bb}$ & $159 \mathrm{Aa}$ \\
\hline $2,5 \times 1(4.000)$ & $57,9 \mathrm{Aa}$ & $46,1 \mathrm{Ab}$ & $0,59 \mathrm{Ba}$ & $0,53 \mathrm{Aa}$ & $1,48 \mathrm{Aa}$ & $0,85 \mathrm{Bb}$ & $2,30 \mathrm{Aa}$ & $1,79 \mathrm{Ba}$ & $141 \mathrm{Aa}$ & $94 \mathrm{Bb}$ \\
\hline $3 \times 0,5(6.667)$ & $45,1 \mathrm{Aa}$ & $48,0 \mathrm{Aa}$ & $0,60 \mathrm{Ba}$ & 0,59Aa & $0,82 \mathrm{Ca}$ & $0,93 \mathrm{Ba}$ & $1,73 \mathrm{Aa}$ & $1,95 \mathrm{Ba}$ & $104 \mathrm{Ba}$ & $115 \mathrm{Aa}$ \\
\hline $3 \times 0,75(4.444)$ & $28,8 \mathrm{Ca}$ & $35,4 \mathrm{Ba}$ & $0,72 \mathrm{Aa}$ & $0,59 \mathrm{Ab}$ & $0,41 \mathrm{Ca}$ & $0,55 \mathrm{Ba}$ & $0,85 \mathrm{Ba}$ & $1,16 \mathrm{Ba}$ & $61 \mathrm{Ca}$ & $68 \mathrm{Ba}$ \\
\hline $3 \times 1(3.333)$ & $36,2 \mathrm{Ba}$ & $42,7 \mathrm{Ba}$ & $0,61 \mathrm{Ba}$ & $0,56 \mathrm{Aa}$ & $0,57 \mathrm{Ca}$ & $0,79 \mathrm{Ba}$ & $1,20 \mathrm{Ba}$ & $1,67 \mathrm{Ba}$ & $73 \mathrm{Ca}$ & $92 \mathrm{Ba}$ \\
\hline $3,5 \times 0,5(5.714)$ & $49,5 \mathrm{Aa}$ & $40,9 \mathrm{Bb}$ & 0,78Aa & $0,57 \mathrm{Ab}$ & $0,98 \mathrm{Ba}$ & $0,72 \mathrm{Ba}$ & $2,10 \mathrm{Aa}$ & $1,52 \mathrm{Ba}$ & $161 \mathrm{Aa}$ & $85 \mathrm{Bb}$ \\
\hline $3,5 \times 0,75(3.810)$ & $45,1 \mathrm{Aa}$ & $49,4 \mathrm{Aa}$ & $0,65 \mathrm{Ba}$ & $0,52 \mathrm{Ab}$ & $0,83 \mathrm{Ca}$ & $1,00 \mathrm{Aa}$ & $1,75 \mathrm{Aa}$ & $2,11 \mathrm{Aa}$ & $114 \mathrm{Ba}$ & $108 \mathrm{Ba}$ \\
\hline $3,5 x 1(2.857)$ & $45,4 \mathrm{Aa}$ & $42,5 \mathrm{Ba}$ & $0,81 \mathrm{Aa}$ & $0,60 \mathrm{Ab}$ & $0,85 \mathrm{Ca}$ & $0,74 \mathrm{Ba}$ & $1,78 \mathrm{Aa}$ & $1,56 \mathrm{Ba}$ & $144 \mathrm{Aa}$ & $93 \mathrm{Bb}$ \\
\hline
\end{tabular}

$\overline{\mathrm{EL}}=$ amostras coletadas na entrelinha do cafeeiro; $\mathrm{PC}=$ amostras coletadas na projeção da copa do cafeeiro. Para cada índice $\mathrm{e}$ profundidade de solo, letras iguais, maiúsculas na coluna e minúsculas na linha, não diferem entre si pelo teste de Scott-Knott $(\mathrm{p} \leq 0,05)$.

Na profundidade de solo 0-0,05 m, os índices de manejo de carbono (IMC) foram superiores a $100 \mathrm{em}$ todos os espaçamentos de plantio, quando as amostras foram coletadas na EL, com valores variando de $107(2 \times 0,5 \mathrm{~m})$ a $251(3 \times 1 \mathrm{~m})$. Ainda na profundidade de 0-0,05 m, na PC, os valores de IMC variaram de $90(2 \times 0,5 \mathrm{~m})$ a $163(2,5 \times 0,5 \mathrm{~m})$, com média de 116 , entre os espaçamentos. Os valores de IMC acima de 100, observados na EL para todos os espaçamentos de plantio, denotam que o manejo implantado nesse local, com maior aporte de resíduos vegetais, contribuiu para aumentar a capacidade de preservação e recuperação dos teores e/ou da qualidade das frações orgânicas presentes no Latossolo sob cultivo de cafeeiro. O maior aporte de resíduos na entrelinha se explica pela maior adição, ao solo, de restos culturais do cafeeiro (folhas, galhos, resíduos da recepa, etc.) e de biomassa de ervas daninhas. Existe a possibilidade também, em função dos resultados positivos que o maior acúmulo de matéria tem sobre a qualidade do solo, de se utilizar nas entrelinhas adubos 
verdes para a produção de palha, cobertura do solo, fixação de $\mathrm{N}$ da atmosfera e ciclagem de nutrientes das camadas mais profundas para a superfície do solo. O uso sistemático de adubos verde-leguminosos pode implicar na possibilidade de obtenção de produtividades adequadas do cafeeiro, sem o uso de adubo nitrogenado mineral. Segundo Amado et al. (2001), o ideal é conciliar o emprego de consórcios de leguminosas com gramíneas, para que os efeitos de suprimento de $\mathrm{N}$, da proteção do solo e da maior permanência da palha, na superfície do solo, sejam potencializados.

É preciso considerar o fato de que as proporções de C-lábil/C-não lábil e, por conseguinte, o IMC, mostramse dependentes do clima e do tipo de solo analisados e que o cálculo do IMC requer ajustes para diferentes tipos de solo e se mostra dependente do método utilizado para determinar os teores de C, em cada fração da MOS (SHANG \& TIESSEN, 1997). Além disso, os padrões de distribuição e os pesos dos teores de $\mathrm{C}$ e dos compartimentos ligados à composição química da matéria orgânica do solo, no cálculo do IMC, podem variar de um local sob cultivo para outro e não refletir os padrões observados em áreas sob vegetação natural, que, quase sempre, é a condição que propicia maior funcionalidade da matriz-solo.

Segundo Tirol-Padre \& Ladha (2004), originalmente, o método de fracionamento do carbono orgânico, baseandose na sua suscetibilidade à oxidação com soluções de $\mathrm{KMnO}_{4}$ de diferentes concentrações (33-333 $\mathrm{mmol} \mathrm{L}^{-1}$ ), foi proposto por Loginow et al. (1987) sobre a premissa de que a decomposição microbiológica da matéria orgânica está associada aos processos de oxidação de caráter enzimático que ocorrem nos solos. Desde então, o C oxidado pelo $\mathrm{KMnO}_{4}$

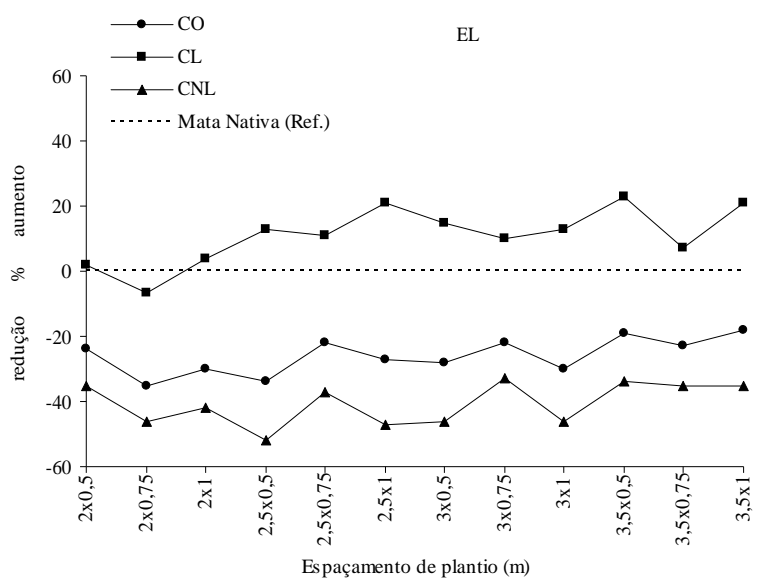

vem sendo utilizado como um índice de C lábil do solo (BLAIR et al., 1995), muito embora a natureza química do C oxidado não seja ainda completamente elucidada. Em solo, as soluções de permanganato oxidam rapidamente compostos orgânicos de baixa biodisponibilidade, em relação aos carboidratos de maior solubilidade, o que indica que esse método de oxidação não discrimina compostos orgânicos lábeis dos não-lábeis. Por tudo isso, o C oxidado pelo $\mathrm{KMnO}_{4}$ não se configura em um índice confiável de determinação do C lábil do solo e, por isso, deve ser referenciado com $\mathrm{C}$ oxidado pelo permanganato, quando utilizado em estudos de caracterização da matéria orgânica do solo (TIROL-PADRE \& LADHA, 2004).

De acordo com Skjemstad et al. (2006), o método de oxidação do $\mathrm{C}$ por permanganato de potássio se mostrou pouco sensível para avaliar ganhos rápidos nos teores de $\mathrm{C}$ orgânico em solos degradados, utilizados para estabelecimento de pastagens; a solução de $\mathrm{KMnO}_{4}$ oxida mais facilmente a lignina e compostos similares a ela, mostrando-se, assim, dependente do tipo de cobertura vegetal do solo. Todas essas considerações e limitações dos métodos utilizados para avaliar o grau de oxidação da MOS se aplicam aos dados apresentados nesse estudo, em função da diversidade de sistemas de plantio e de manejo da lavoura cafeeira avaliados e pelo fato de o $\mathrm{KMnO}_{4}$ não poder ser aplicado de forma indiscriminada para avaliar a biodisponibilidade de frações orgânicas presentes em áreas sob influência de diferentes tipos de vegetação, solos e práticas de manejo (SHANG \& TIESSEN, 1997; SKJEMSTAD et al., 2006).

Apresentam-se na Figura 1 os aumentos e reduções porcentuais nos teores de carbono de diferentes frações das

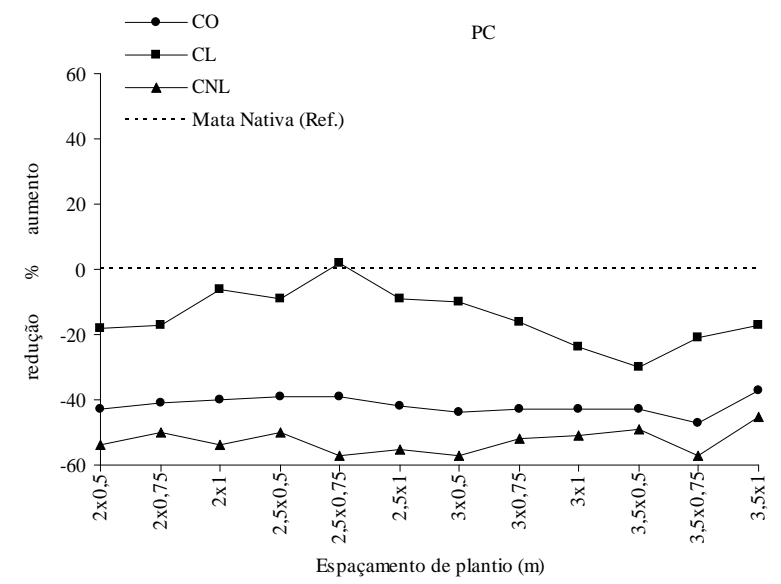

Figura 1 -Aumentos e reduções porcentuais de teores de C em frações da matéria orgânica de Latossolo Vermelho distroférrico típico cultivado com cafeeiro em diferentes espaçamentos de plantio, em relação ao sistema referência (mata). EL: amostras coletadas na entrelinha; PC: amostras coletadas na projeção da copa. $\mathrm{CO}=$ carbono orgânico do solo (combustão via úmida por dicromato); $\mathrm{C}_{\mathrm{L}}=$ carbono lábil; $\mathrm{C}_{\mathrm{NL}}=$ carbono não-lábil. Os porcentuais de $\mathrm{CO}, \mathrm{C}_{\mathrm{L}}$ e $\mathrm{C}_{\mathrm{NL}}$, nos diferentes espaçamentos de plantio do cafeeiro, representam a média dos valores obtidos nas profundidades de solo de 0-0,05 e 0-0,1 m. 
amostras coletadas nos diferentes pontos de coleta (EL e PC) e espaçamentos de plantio do cafeeiro. Os atributos do solo na área de mata foram utilizados como referência. A amplitude de variação dos teores de $\mathrm{C}$ lábil, em geral, foi maior do que a verificada para o $\mathrm{CO}$ e carbono não-lábil $\left(\mathrm{C}_{\mathrm{NL}}\right)$ do solo. Os resultados apresentados permitem inferir que o $\mathrm{C}_{\mathrm{L}}$ pode apresentar variações positivas (aumentos) e negativas (decréscimos) num menor intervalo de tempo que o $\mathrm{CO}$ e o $\mathrm{C}_{\mathrm{NL}}$ sendo, por esse motivo, um índice importante para avaliar mudanças na MOS, em função da adoção de diferentes sistemas de cultivo de cafeeiro. De fato, alterações positivas (aumentos) só foram notadas para os teores de C lábil, na entrelinha do cafeeiro sob influência de diferentes espaçamentos de plantio, à exceção do plantio nas dimensões de $2 \times 0,75 \mathrm{~m}$. Nas amostras coletadas na projeção da copa, em relação à área de mata, foram notadas diminuições dos teores de $\mathrm{C}$ nas frações orgânicas. Em relação aos teores de $\mathrm{C}$ nãolábil e CO, as reduções verificadas para as amostras de solo da projeção da copa foram mais acentuadas do que as notadas para as amostras coletadas na entrelinha do cafeeiro.

\section{CONCLUSÕES}

Na camada superficial do solo $(0-0,05 \mathrm{~m})$, os teores de C lábil na entrelinha são, em geral, maiores do que os observados nas amostras coletadas na projeção da copa do cafeeiro.

O índice de manejo do carbono (IMC) variou de 61 a 251 e os maiores valores de IMC foram observados, em geral, na entrelinha, onde a largura de rua do cafeeiro era de 3 metros.

A amplitude de variação dos teores de $\mathrm{C}$, nas frações orgânicas, obedece à seguinte ordem decrescente: $\mathrm{C}$ lábil> não-lábil > C orgânico, o que indica o maior potencial do C lábil em avaliar o impacto de diferentes sistemas de adensamento e manejo do cafeeiro sobre os compartimentos de matéria orgânica do solo.

Os teores de C lábil e C não-lábil não são influenciados pelo estande de plantas do cafeeiro, mas se mostram dependentes da largura de rua do cafeeiro, da profundidade e do local de coleta das amostras de solo.

\section{AGRADECIMENTOS}

À equipe da Estação Experimental da EpamigMachado, MG, em especial ao senhor Gilmar José Cereda, pela ajuda na coleta das amostras e na condução e manutenção do experimento. Ao Consórcio Brasileiro de Pesquisa e Desenvolvimento/Café, à Embrapa Café (Projeto 19.2003.133.03) e à FAPEMIG (EDT 2222/2003), pela concessão de fomentos utilizados no custeio das ações de pesquisa.

\section{REFERÊNCIAS BIBLIOGRÁFICAS}

AMADO, T. J. C.; BAYER, C.; ELTZ, F. L. P.; BRUM, A. C. R. Potencial de plantas de cobertura em acumular carbono e nitrogênio no solo no plantio direto e a melhoria da qualidade ambiental. Revista Brasileira de Ciência do Solo, Viçosa, v. 25, p. 189-197, 2001.

ANDRADE, C. A.; OLIVEIRA, C. de; CERRI, C. C. Qualidade da matéria orgânica e estoques de carbono e nitrogênio em Latossolo tratado com biossólido e cultivado com eucalipto. Revista Brasileira de Ciência do Solo, Viçosa, v. 29, n. 5, p. 803-816, 2005.

ASSOCIAÇÃO BRASILEIRA DAS INDÚSTRIAS DE CAFÉ. ABIC. Disponível em: 〈http://www.abic.com.br〉. Acesso em: 8 mar. 2006.

BAYER, C.; BERTOL, I. Características químicas de um Cambissolo húmico afetadas por sistemas de preparo, com ênfase à matéria orgânica. Revista Brasileira de Ciência do Solo, Viçosa, v. 23, p. 687-694, 1999.

BLAIR, G. J.; LEFROY, R. D. B.; LISLE, L. Soil carbon fractions based on their degree of oxidation, and the development of a carbon management index for agricultural systems. Australian Journal of Agricultural Research, Victoria, v. 46, p. 1459-1466, 1995.

CHAN, K. Y.; BOWMAN, A.; OATES, A. Oxidizible organic carbon fractions and soil quality changes in an paleustalf under different pasture leys. Soil Science, Baltimore, v. 166, n. 1, p. 61-67, 2001.

CIOTTA, M. N.; BAYER, C.; ERNANI, P. R.; FONTOURA, S. M. V.; ALBUQUERQUE, J. A.; WOBETO, C. Acidificação de um latossolo sob plantio direto. Revista Brasileira de Ciência do Solo, Viçosa, v. 26, p. 1055-1064, 2002.

FREITAS, A. G.; MATOS, E. S.; MENDONÇA, E. S. Matéria orgânica e estabilidade de agregados em diferentes sistemas de adubação. In: FERTBIO, 2004, Lages. Anais... Lages: UDESC/SBCS, 2004. CD-ROM.

GUERTAL, E. A.; ECKERT, D. J.; TRAINA, S. J.; LOGAN, T. J. Differential phosphorus retention in soil profiles under no-till crop production. Soil Science Society of America Journal, Madison, v. 55, p. 410-413, 1991.

LOGINOW, W.; WISNIEWSKI, W.; GONET, S. S.; CIESCINSKA, B. Fractionation of organic $\mathrm{C}$ based on susceptibility to oxidation. Polish Journal of Soil Science, [S.1.], v. 20, p. 47-52, 1987. 
MIELNICZUK, J.; BAYER, C.; VEZZANI, F. M.; LOVATO, T.; FERNANDES, F. F.; DEBARBA, L. Manejo de solo e culturas e sua relação com os estoques de carbono e nitrogênio do solo. Tópicos em Ciência do Solo, Viçosa, v. 3, p. 209-248, 2003.

PAVAN, M. A.; CHAVES, J. C. D. Alterações nas frações de fósforo no solo associadas com a densidade populacional de cafeeiros. Revista Brasileira de Ciência do Solo, Campinas, v. 20, p. 251-256, 1996.

PAVAN, M. A.; CHAVES, J. C. D.; SIQUEIRA, R.; ANDROCIOLI FILHO, A.; COLOZZI FILHO, A.; BALOTA, E. L. High coffee population density to improve fertility of an oxisol. Pesquisa Agropecuária Brasileira, Brasília, v. 34, n. 3, p. 459-465, 1999.

PRESTON, C. M. Applications of NMR to soil organic matter analysis: history and prospects. Soil Science, Baltimore, v. 161, n. 3, p. 144-166, 1996.

SCHERER, E. E.; BALDISSERA, I. T.; NESI, C. N. Propriedades químicas de um Latossolo Vermelho sob plantio direto e adubação com esterco de suínos. Revista Brasileira de Ciência do Solo, Campinas, v. 31, n. 1, p. 123-131, 2007.

SHANG, C.; TIESSEN, H. Organic matter lability in a tropical oxisol: evidence from shifting cultivation, chemical oxidation, particle size, density and magnetic fractionations. Soil Science, Baltimore, v. 162, n. 11, p. 795-807, 1997.

SILVA, C. A.; ANDERSON, S. J.; VALE, F. R. Carbono, nitrogênio e enxofre em frações granulométricas de dois Latossolos submetidos à calagem e adubação fosfatada. Revista Brasileira de Ciência do Solo, Viçosa, v. 23, p. 593-602, 1999.
SILVA, J. E.; LEMAINSKI, J.; RESCK, D. V. S. Perdas de matéria orgânica e suas relações com a capacidade de troca catiônica em solos da região de cerrados do oeste baiano. Revista Brasileira de Ciência do Solo, Campinas, v. 18, n. 3, p. 541-547, 1994.

SKJEMSTAD, J. O.; SWIFT, R. S.; McGOWAN, J. A. Comparison of the particulate organic carbon and permanganate oxidation methods for estimating labile soil organic carbon. Australian Journal of Soil Research, Victoria, v. 44, n. 3, p. 255-263, 2006.

STEVENSON, F. J. Humus chemistry: genesis, composition, reactions. 2. ed. New York: J. Wiley \& Sons, 1994. $496 \mathrm{p}$.

TIROL-PADRE, A.; LADHA, J. K. Assessing the reliability of permanganate-oxidizable carbon as an index of soil labile carbon. Soil Science Society of America Journal, Madison, v. 68, p. 969-978, 2004.

WALKLEY, A.; BLACK, A. An examination of the Degtjareff method for determining soil organic matter and a proposed modification of the chromic acid titration method. Soil Science, Baltimore, v. 37, p. 29-38, 1934.

YEOMANS, J. C.; BREMNER, J. M. A rapid and precise method for routine determination of organic carbon in soil. Communications in Soil Science and Plant Analysis, New York, v. 19, p. 1467-1476, 1988.

ZECH, W.; HAUMAIER, L.; HEMPFLING, R. Ecological aspects of soil organic matter in tropical land use. In: McCARTHY, P.; CLAPP, C. E.; MALCOM, R. L.; BLOOM, P. R. (Eds.). Humic substances in soil and crop sciences: selecting readings. Madison: ASA/SSSA, 1990. p. 187-220. 